\title{
Application of 'smart' amine donors for rapid screening and scale-up of transaminase-mediated biotransformations
}

Andrew Gomm, ${ }^{[a]}\left[{ }^{[\neq]}\right.$Stylianos Grigoriou, ${ }^{[a]}{ }^{[\neq]}$Christopher Peel,,${ }^{[a]}$ James Ryan, ${ }^{[a]}$ Nafees Mujtaba, ${ }^{[a]}$ Thomas Clarke, ${ }^{[a]}$ Evelina Kulcinskaja ${ }^{[a]}$ and Elaine O'Reilly* [a] 
THE 'SMART' AMINE DONORS O-XYLYLENEDIAMINE AND CADAVERINE WERE EMPLOYED FOR THE RAPID SCREENING OF A LARGE KETONE LIBRARY AND SUBSEQUENT PREPARATIVESCALE SYNTHESIS OF SELECTED COMPOUNDS USING THE COMMERCIALLY AVAILABLE AMINE TRANSAMINASE, ATA256. THE METHODOLOGY ENABLES BOTH SCREENING AND PREPARATIVE-SCALE BIOTRANSFORMATIONS TO BE PERFORMED WITH A SINGLE ENZYME AND SIMPLIFIES THE GENERATION OF SP3-RICH SMALL MOLECULE LIBRARIES.

Chiral amines are ubiquitous in pharmaceutical drugs, bioactive natural products and privileged scaffolds. ${ }^{[1-2]}$ Asymmetric introduction of amines into small molecule libraries represents an attractive way of increasing their complexity. ${ }^{[3-5]}$ It has been demonstrated that ' $\mathrm{sp}^{3}$-rich' compounds possessing one or more stereocentres have favourable properties for drug discovery processes. ${ }^{[6-7]}$ In order to produce diverse libraries of optically pure chiral amines, robust strategies for their preparation must be developed.

Current methods for asymmetric amine synthesis typically rely on chiral auxiliaries or precious metal catalysts and often require harsh reaction conditions (high temperatures, pressures etc). ${ }^{[8]}$ The application of enzymes for their synthesis represents a sustainable alternative, as biotransformations are normally carried out under mild conditions and often display superior regio- and stereoselectivity to the traditional chemical approaches.

Amine transaminase (ATA) enzymes have emerged as powerful catalysts for the production of chiral amines, with many impressive examples of their application present in the scientific literature. ${ }^{[9-12]}$ ATAs are pyridoxal-5-phosphatedependent (PLP-dependent) and can catalyse the reversible formation of chiral amines from pro-chiral ketones using a suitable sacrificial amine donor. The reversibility of this transformation means that efficient methods to displace the often challenging reaction equilibrium are necessary to achieve high conversion of substrate to product.

Commonly employed strategies include the use of a large excess of amine donor coupled with in situ co-product removal or enzymatic degradation of the reaction byproduct. $[9,10,13-16]$ We previously demonstrated that orthoxylyenediamine can be used to effectively displace the reaction equilibrium of some of the most challenging substrates, using near stoichiometric equivalents of the 'smart' diamine donor. ${ }^{[17]}$ Additionally, the co-products formed upon transamination with this donor are highly coloured, suggesting that it could be employed for highthroughput screening of both large enzyme and ketone libraries. We have also extended this methodology to other low-cost diamine donors, including cadaverine. ${ }^{[18]}$ The efficient conversion achieved using these diamines can be attributed to the spontaneous cyclisation and subsequent oligimerization/polymerization of the co-products, which effectively displaces the reaction equilibrium. This methodology has since been shown to be effective with other
ATAs and importantly, with other low-cost, bulk diamine donors. ${ }^{[19]}$ The development of biocatalytic approaches for the synthesis of chiral amines that exploit 'smart' low-cost diamine donors, in place of IPA coupled with complex coproduct removal, is of great interest.

We have now demonstrated that $o$-xylylenediamine can be employed for the rapid screening of a large library of commercially available ketones and aldehydes, enabling expedient identification of transaminase substrates. Based on this high-throughput screening data, a selection of ketones were converted to the corresponding chiral primary amines on a preparative-scale, using cadaverine. The approach demonstrates that these two smart donors can be used in combination for efficient screening and subsequent scale-up, using lower cost diamine donors.

A library containing 400 ketones/aldehydes was supplied by our industrial partner, Key Organics. Compounds within this library were structurally diverse and many of the corresponding amines were of commercial interest. Screening was conducted in 96-well plates and results analysed after 1 and 24 hours (figure 1). The formation of a dark precipitate in the wells enabled qualitative identification of enzyme substrates.

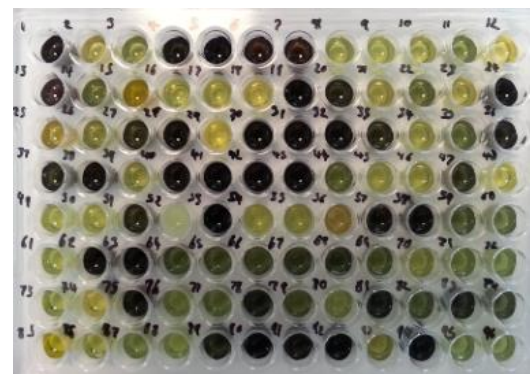

Figure 1. Representative assay plate showing the screening of a ketone library using $o$-xylylenediamine. Wells containing dark precipitate indicate ATA activity.

Analysis of the assay data (figure. 2) revealed that smaller, low molecular weight compounds (<225 Da) are readily accepted by commercially available ATA 256; an enzyme developed by Codexis. ${ }^{[20]}$ This data demonstrates that in a library of diverse compounds, larger molecules are less likely to be accepted. However, molecular weight and size are not the only important factors and electronic effects, as well as the nature of the substituents on the ketone are also extremely relevant. As expected, the data showed that alpha aryl-substituted methyl ketones were less amenable to transamination compared to those with an alkyl substituent (figure. 3). Cyclic ketones were well tolerated whereas $\alpha, \beta$ unsaturated ketones were poorer substrates. ${ }^{[21]}$

Several ketones were then selected for quantitative analysis (Figure 3 ) based on our interest in producing the corresponding chiral amines on a preparative scale. The amines selected were either not commercially available, were extremely expensive or were difficult to produce using traditional chemical approaches. Analytical scale reactions 


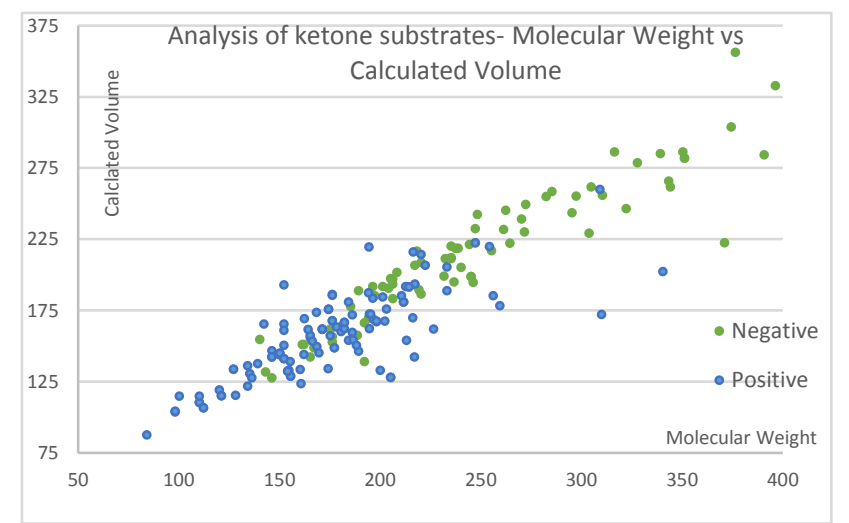

Figure 2. Characterisation of ketones used in the library screening and a plot of their molecular weight vs volume. ${ }^{[22,22]}$

were performed using our recently reported diamine donor cadaverine $^{[17]}$ and compared to transformations using isopropylamine (the current industry standard amine donor). As expected, reactions employing cadaverine outperformed isopropylamine in almost all cases (figure 3). Using 3 equivalents of cadaverine was sufficient to achieve a $98 \%$ conversion of challenging ketone $\mathbf{1}$ to the corresponding amine. A conversion of only $70 \%$ was achieved using IPA at the same concentration. The diamine donor also significantly outperformed IPA in the biotransformations involving the low molecular weight ketones, 5-8. These ketones (5-8) have particularly low boiling points and therefore using high concentrations of IPA in combination with in situ acetone coproduct removal is likely to be challenging, as the volatile ketone starting material would also be evaporated under these conditions. For this reason, we choose to demonstrate the utility of the 'smart' amine donor, cadaverine, and by extension similar low-cost diamine donors, by preforming reparative-scale biotransformations with ketones $\mathbf{1}$ and 12 and volatile ketones 6-8.

Initial studies showed that using DMSO as a co-solvent was likely to complicate chromatographic purification when reactions were performed on a preparative scale. A variety of other co-solvents were screened and biotransformations containing methanol and ethanol reached similar conversions to those with DMSO, indicating that these were good alternative solvents. Acetone was the only co-solvent that gave poor conversions, with some of the biotransformations being completely inhibited (see supplementary data).

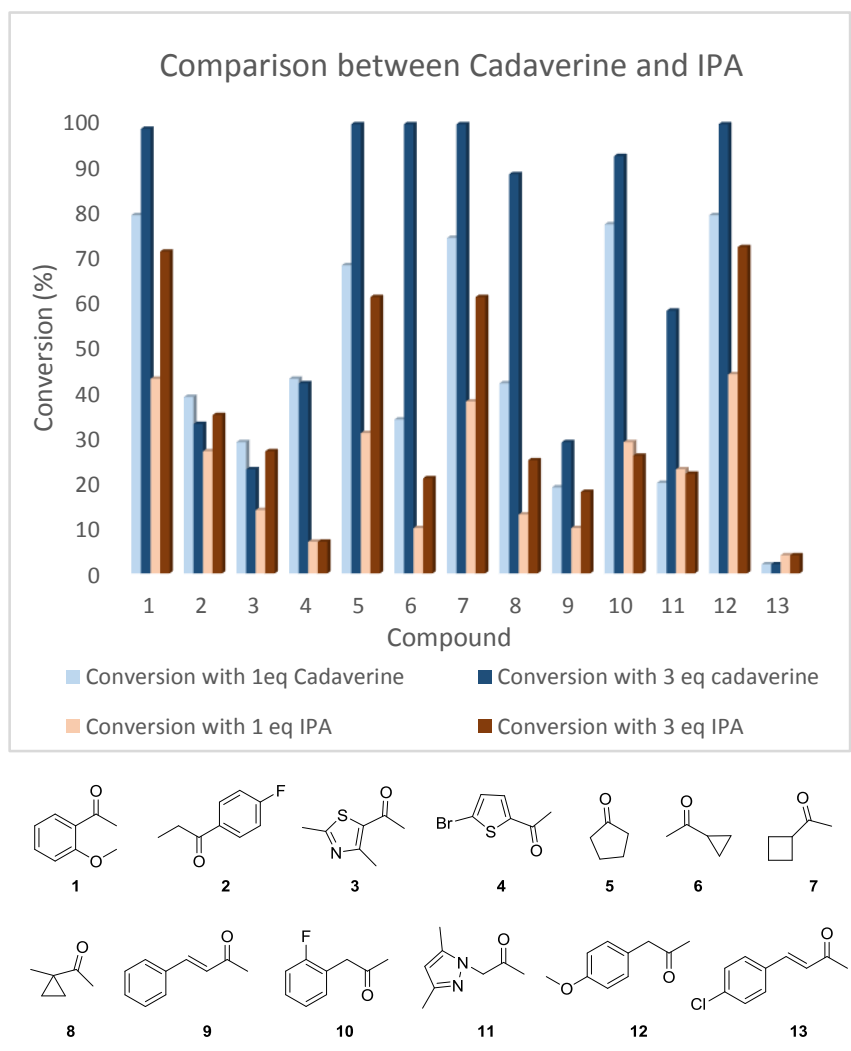

Figure 3: Analytical scale biotransformations with a selection of ketone substrates 1-13, utilising either 1 or 3 equivalents of the amine donors, cadaverine or IPA. Conversions of compounds 1-13 $(20 \mathrm{mM})$ to the corresponding amine were carried out using ATA256 (2.5 mg/ml), PLP (1 $\mathrm{mM})$ and cadaverine or IPA $(20 \mathrm{mM})$. Conversions are an average of three replicates.

Preparative biotransformations were carried out with ketones 6-8 in HEPES buffer at $\mathrm{pH} 10.9$, using DMSO as a co-solvent and were purified by distillation (see ESI for further details). All reactions proceeded with excellent ee (>99\%), which was established by the synthesis and NMR analysis of the corresponding Mosher's acid chlorides (see supporting information for details). ${ }^{[2]}$ Compounds $\mathbf{1}$ and 12 were extracted and purified via column chromatography in $70 \%$ and $39 \%$ yield respectively. In all cases, the compounds were of sufficient purity for commercialisation.

The synthesis and application of ' $\mathrm{sp}^{3}$-rich' fragments remains highly important in modern drug-discovery processes and it is expected that this methodology can provide synthetic and medicinal chemists with a facile route for the synthesis of chiral amine fragments. There is increasing interest from industry in the development of sustainable, bio-derived chiral molecules and the use of commercially available enzymes and mild reaction conditions make this approach attractive for accessing these synthetically challenging compounds. Work is on-going to directly evolve new enzymes that possess a broader 
substrate scope and that can utilise 'smart' amine donors. This will expand the use of the biocatalytic toolbox in modern synthesis.

\section{Acknowledgements}

We thank our industrial partners, Key Organics, for supplying the compound library and BIOCATNET, a BBSRC NIBB (BB/L013649/1) for proof-of-concept funding. Research leading to these results has also received funding from the BBSRC (BB/M021947/1) and Royal Society Research Grant (RG150134). The authors gratefully acknowledge the BBSRC/EPSRC Synthetic Biology Research Centre, Nottingham and the University of Nottingham for the financial support provided to SG. We also acknowledge funding from the UoN to support $A G$, funding from the UK Catalysis Hub for supporting JR and funding from the EPSRC Centre for Doctoral Training in Sustainable Chemistry for supporting CP and TC.

[1] D. J. Newman, G. M. J. Cragg, Nat. Prod. 2012 75, 311.

[2] M. E. Welsch, S. A. Snyder, B. R. Stockwell, Curr. Opin. Chem. Biol. 201014,347

[3] F. Lovering, J. Bikker and C. Humblet, J. Med. Chem., 200952 6752-6756.

[4] P. Piras and C. Roussel, J. Pharm. Biomed. Anal., 2008 46, 839847.

[5] W. R. J. D. Galloway, A. Isidro-Llobet and D. R. Spring, Nat. Commun., 2011 1, 1-13.

[6] M. Feher and J. M. Schmidt, J. Chem. Inf. Comput. Sci., 200343 218-227.

[7] P. A. Clemons, N. E. Bodycombe, H. A. Carrinski, J. A. Wilson, A. F. Shamji, B. K. Wagner, A. N. Koehler and S. L. Schreiber, Proc. Natl. Acad. Sci. U. S. A., 2010 107, 18787-18792.

[8] T. C. Nugent and M. El-Shazly, Adv. Synth. Catal., 2010 352, 753819

[9] C. K. Savile et al., Science 2010 329, 305

[10] Andrew Gomm and Elaine O'Reilly, Curr Opin Chem Biol. 2018 43,106-112.

[11] F. Guo and P Berglund, Green Chem. 2017, 19, 333-360.

[12] I. Slabu, J. L. Galman, R. C. Lloyd and N. J. Turner, Green Chem. 2017, 7, 8263-8284.

[13] T. Bo, G. Rehn, C. Grey and P. Adlercreutz, Org. Process Res. Dev., 2015 19, 793-799.

[14] M. D. Truppo, J. D. Rozzell and N. J. Turner, Org. Process Res. Dev., 2010 14, 234-237.

(n)

J. S. Shin, B. G. Kim, Biotechnol. Bioeng. 1999 65, 206-211.

M. D. Truppo, J. D. Rozzell, J. C. Moore, N. J. Turner, Org.Biomol. Chem. 2009 7, 395-398.

A. P. Green, N. J. Turner and E. O'Reilly, Angew. Chemie - Int. Ed., 2014 53, 10714-10717.

A. Gomm, W. Lewis, A. P. Green and E. O'Reilly, Chem. - A Eur. J., 2016, 22, 12692-12695.

J. L. Galman, I. Slabu, N. J. Weise, C. Iglesias, F. Parmeggiani, R. C. Lloyd, N. J. Turner, Green Chem., 2017 9, 285-288.

0] The sequence for ATA256 can be found in the following patent. Crowe et. al. Chemical processes for preparing spiroindolones and intermediates thereof. US Patent US 2015/0045562 A1, filed 22 March 2013 and issued 12 February 2015.

Minor modification of the $o$-xylylenediamine assay to use 0.15 $\mathrm{mg} / \mathrm{mL}$ of commercially available enzyme enzyme and leaving for 3 hours instead of 24 hours gave enabled accurate prediction of substrates which underwent facile transamination. (see Supplementary Data for more information).

Volume calculated with Molinspiration

(http://www.molinspiration.com), the tool implemented in virtual computational chemistry laboratories (VCCLAB), http://www.vcclab.org.

[23] I. V. Tetko, J. Gasteiger, R. Todeschini, A. Mauri, D. Livingstone, P. Ertl, V. A. Palyulin, E. V. Radchenko, N. S. Zefirov, A. S. Makarenko, V. Y. Tanchuk and V. V. Prokopenko, J. Comput.-Aided Mol. Des., 2005, 19, 453. 
